JiR RIN

Volume 6, No. 4 October - December 2020 www.jrmi.pk

September 20, 2020

Accepted

October 25, 2020

Author Information

Dr. Wajeeha Qayyum Senior Registrar

Medicine and Allied

Rehman Medical Institute,

Peshawar, Khyber

Pakhtunkhwa, Pakistan.

(Corresponding Author)

Email

drwajeeha13@yahoo.com

Dr. Mohammad Aman

Trainee Medical Officer

Pulmonology, Rehman

Medical Institute, Peshawar.

Dr. Asad Khalil

House Officer

Rehman Medical Institute,

Peshawar

Dr. Mohammad Jawad

Lecturer

Family Medicine

Khyber Medical University

Peshawar.

Dr. Seema Ashraf

Assistant Professor

Community Medicine

Rehman Medical College,

Peshawar.

Citation: Qayyum W, Aman M, Khalil A, Jawad M, Ashraf S. Psychological status of healthcare workers during COVID-19 pandemic: a single center observational study from Peshawar. J Rehman Med Inst. 2020 OctDec;6(4):3-6.

\title{
Psychological status of healthcare workers during COVID-19 pandemic: a single center observational study from Peshawar
}

\author{
Wajeeha Qayyum, Mohammad Aman, Asad Khalil, Mohammad Jawad, Seema Ashraf
}

\section{ABSTRACT}

Introduction: Infectious pandemics are a significan health risk to the general population, but much more so for the frontline health care workers tending patients at different locations. The increased workload, stress, fear, and anxiety are the burdens to be coped with by health professionals through resort to resiliency and other mechanisms.

Objective: To determine the magnitude of depression / anxiety and factors associated with it in health care workers of a tertiary care hospital during the COVID-19 pandemic in Peshawar, Khyber Pakhtunkhwa, Pakistan

Materials \& Methods: This cross sectional study was conducted at Rehman Medical Institute, Peshawar after approval from Rehman Medical Institute Research Ethics Committee. A structured questionnaire consisting of two sections was distributed by convenience sampling to health care workers in the hospital, the first part containing demographic information, and the second part consisting of Hospital Anxiety and Depression Scale (HADS) comprised of 14 scales to assess anxiety and depression. Scoring for Normal, Borderline Abnormal, and Definitive Disorder was done as per standard criteria. Data were analyzed for descriptive statistics by SPSS 22 .

Results: Total 327 participants were included in the study, having mean age of $30.19 \pm 7.82$ years, of which $230(70.3 \%)$ were males. The majority of health care workers 226(69.1\%) were suffering from disturbed mental status either in the form of Anxiety (19.9\%), Depression $(8 \%)$ or Mixed Disorder (41.3\%). Factors associated with high disease frequency were female gender, age group 21-30 years, nursing staff, workers of ICU and Hostel residence ( $\mathrm{p}$ value $<0.05$ ).

Conclusion: COVID-19 related psychological disturbances significantly affected healthcare staff of a tertiary care hospital of Peshawar, being more evident in females, younger age groups, nurses, and ICU staff on duty.

Keywords: Coronavirus; Depression; Anxiety; Health Care Workers; Pandemic.

The authors declared no conflict of interest. All authors contributed substantially to the planning of research, data collection, data analysis, and write-up of the article, and agreed to be accountable for all aspects of the work.

\section{INTRODUCTION}

The COVID-19 pandemic that started towards the end of 2019 now seems to be fading in majority of regions. However, it has left multiple impacts especially in the form of Post-Traumatic Stress Disorders (PTSD). Literature on previous pandemics highlighted significant impact of it on mental health of population. ${ }^{1}$ The risk is doubled on Health Care Workers (HCWs) due to their close and repeated contact with infectious patients in hospitals, quarantined period after exposure, repeated need for screening and witnessing their colleagues contracting the infection from hospitals. All these factors make HCWs prone to greater stress related mental disturbances than normal people in the community. ${ }^{2}$ As this pandemic was more contagious and had more global spread than SARS CoV-1, so it has brought challenges and threatens the public health security around the globe. ${ }^{3}$ During this pandemic, due to over flooded hospital with serious and infected patients, inadequate supply of Personal protective Equipment (PPE) and limited recourses cast the major stress on HCWs, both physical and psychological. $^{4}$

A significant number of HCWs got infected and few died in the line of duty at forefronts providing medical care to patients. According to report submitted by World Health Organization (WHO), HCWs accounted for $21 \%$ cases of COVID-19 globally. ${ }^{5}$ Data from Italy, China, United States of America, Italy, Spain, France and China showed that $15-18 \%$ of HCWs acquired COVID-19 infections. ${ }^{6}$ Newer analysis by Amnesty International has revealed that $7000 \mathrm{HCWs}$ lost their lives after contracting COVID-19. The highest figures are being reported from Mexico where HCWs deaths exceeded 1300 followed by USA and Brazil. ${ }^{7}$ In Pakistan, by June 30, 5367 HCWs had contracted the viral infection and 58 died. ${ }^{8}$

In a systematic review consisting of above 33000 participants from 13 studies described a pooled prevalence of anxiety as $23.2 \%$ and depression as $22.8 \% .^{9}$ Data from Pakistan reported that $42 \%$ HCWs suffered from moderate and $26 \%$ from severe psychological distress during this pandemic. ${ }^{10}$ However, the pooled prevalence of depression was estimated to be $23 \%$. $^{9}$ 
The rapid, life threatening and contagious disease course had led to extraordinary amounts of pressure on HCWs. The fear of nosocomial transmission, and in a resource limited situation, the need to make ethically difficult decisions regarding patients care may cast significant effects on their physical and mental wellbeing. ${ }^{9}$ Therefore, they are at risk to suffer from complex emotional and psychological distress. ${ }^{10}$ The importance of this issue lies in the fact that the mental health problems of HCWs would compromise their attention and decision making ability in emergency situations consequently ending up in medical errors that ultimately putting patients at risk. ${ }^{11}$ Moreover, for their own selves, this distress can affect lifelong mental being. ${ }^{12}$ Hence, it is important to assess mental health status of HCWs working in COVID-19 epidemic periodically to identify the sufferers at early stage and also to modify the policies to reduce the incidence of it. $^{13}$

In Khyber Pakhtunkhwa (KP) province, as the percentage of infected healthcare workers is higher as compared to other provinces, it is likely that psychological stress and emotional disturbances in HCWs may have high magnitude. To our best knowledge no study has focused on mental status of HCW from this area. We tried to evaluate the depression/anxiety status of $\mathrm{HCW}$ working in tertiary care center of Peshawar.

The objective of this study was to determine the magnitude of depression / anxiety and to look for factors associated with it in health care workers of a tertiary care hospital of Peshawar, KP.

\section{MATERIALS \& METHODS}

This cross-sectional descriptive study was done at Rehman Medical Institute (RMI), Peshawar in July 2020, after taking approval from Rehman Medical Institute Research Ethics Committee. A structured Questionnaire consisting of two sections was distributed by convenience sampling to health care workers in the hospital. The first part of questionnaire contained demographic information such as age, gender, designation, place of duty and residence. The second part consisted of Hospital Anxiety and Depression Scale (HADS), comprising of 14 scales to assess anxiety and depression. Score was 0-21 for each subscale. AS per standard criteria, score 0-7 is considered Normal, 8-10 is categorized as Borderline Abnormal (borderline case) and 11-21 indicates Definitive Disorder. In the present study, the medical doctors of all levels, nurses, nursing internees, and respiratory therapists were eligible for inclusion; excluded were those unwilling to participate, could not understand English language, health care assistants like pharmacists, physiotherapist etc., and other hospital staff including ward boys, management / administrative personnel. The data were collected over the period of one week in the peak of pandemic from July1 to July7, 2020.

All the questionnaires were reviewed and total score of HADS calculated; participants were classified into Anxiety, Depression, Both or None disorder. Anxiety and Depression were further classified as Borderline or Definitive disorder depending upon the score.

Data were analyzed in SPSS 21. Mean \pm SD, percentages and frequencies were calculated of quantitative and qualitative variables respectively. Association of disorder was noted through
Chi Square test with age groups, gender, place of duty, designation, and hostel residence $p$ value $\leq 0.05$ was considered statistically significant.

\section{RESULTS}

Total 327 participants were included in the study. Mean age was $30.19 \pm 7.82$ years, and $230(70.3 \%)$ were males. Details regarding study population are provided in Table 1 .

Table 1: Characteristics of health care personnel $(n=327)$.

\begin{tabular}{|l|l|c|}
\hline \multicolumn{2}{|c|}{ Characteristics } & $\begin{array}{c}\text { Frequency } \\
(\%)\end{array}$ \\
\hline \multirow{4}{*}{ Designation } & TMO & $101(30.9)$ \\
\cline { 2 - 3 } & MO & $50(15.3)$ \\
\cline { 2 - 3 } & HO & $58(17.7)$ \\
\cline { 2 - 3 } & Nurse & $44(13.5)$ \\
\cline { 2 - 3 } & Nursing internee & $19(05.8)$ \\
\cline { 2 - 3 } & Respiratory Therapist & $07(02.1)$ \\
\cline { 2 - 3 } & Registrar & $30(09.2)$ \\
\cline { 2 - 3 } & Consultant & $18(05.5)$ \\
\hline \multirow{5}{*}{ Place of duty } & Medicine & $98(30.0)$ \\
\cline { 2 - 3 } & Surgery & $42(12.8)$ \\
\cline { 2 - 3 } & Gynecology & $31(09.5)$ \\
\cline { 2 - 3 } & Peads & $35(10.7)$ \\
\cline { 2 - 3 } & Cardiac Surgery & $33(10.1)$ \\
\cline { 2 - 3 } & Anesthesia & $21(06.4)$ \\
\cline { 2 - 3 } & ICU & $41(12.5)$ \\
\cline { 2 - 3 } & Covid Ward & $26(08.0)$ \\
\hline \multirow{5}{*}{ Residence } & Hostel & $110(33.6)$ \\
\cline { 2 - 3 } & Home & $217(66.4)$ \\
\hline
\end{tabular}

A majority of $226(69.1 \%$ ) health care workers were suffering from disturbed mental status either in the form of Anxiety, Depression or Both. Figure 1 presents the details in this regard.

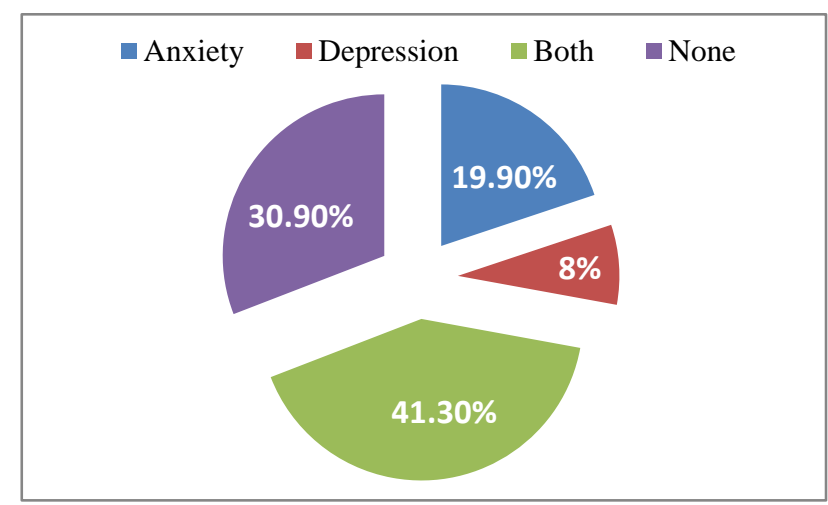

Figure 1: Distribution of disturbed mental status among health care personnel $(n=327)$.

Among health care workers suffering from Depression, 91 (67.4\%) had Borderline Depression, and 44(32.6\%) had Definitive Depression. Among the ones with Anxiety Disorder 77(57\%) had Borderline Anxiety, while 58(43\%) fell into category of Definitive Anxiety disorder. 
To see the association of disturbed mental state with different factors, subjects were stratified by age groups. Furthermore, health care workers were categorized as junior doctors (TMO,
MO, HO), senior doctors (Registrar, Consultants) and nursing staff including nurses, internees, and respiratory therapists. Table 2 presents the details in this regard.

Table2: Factors associated with different disorders in health care personnel $(n=327)$.

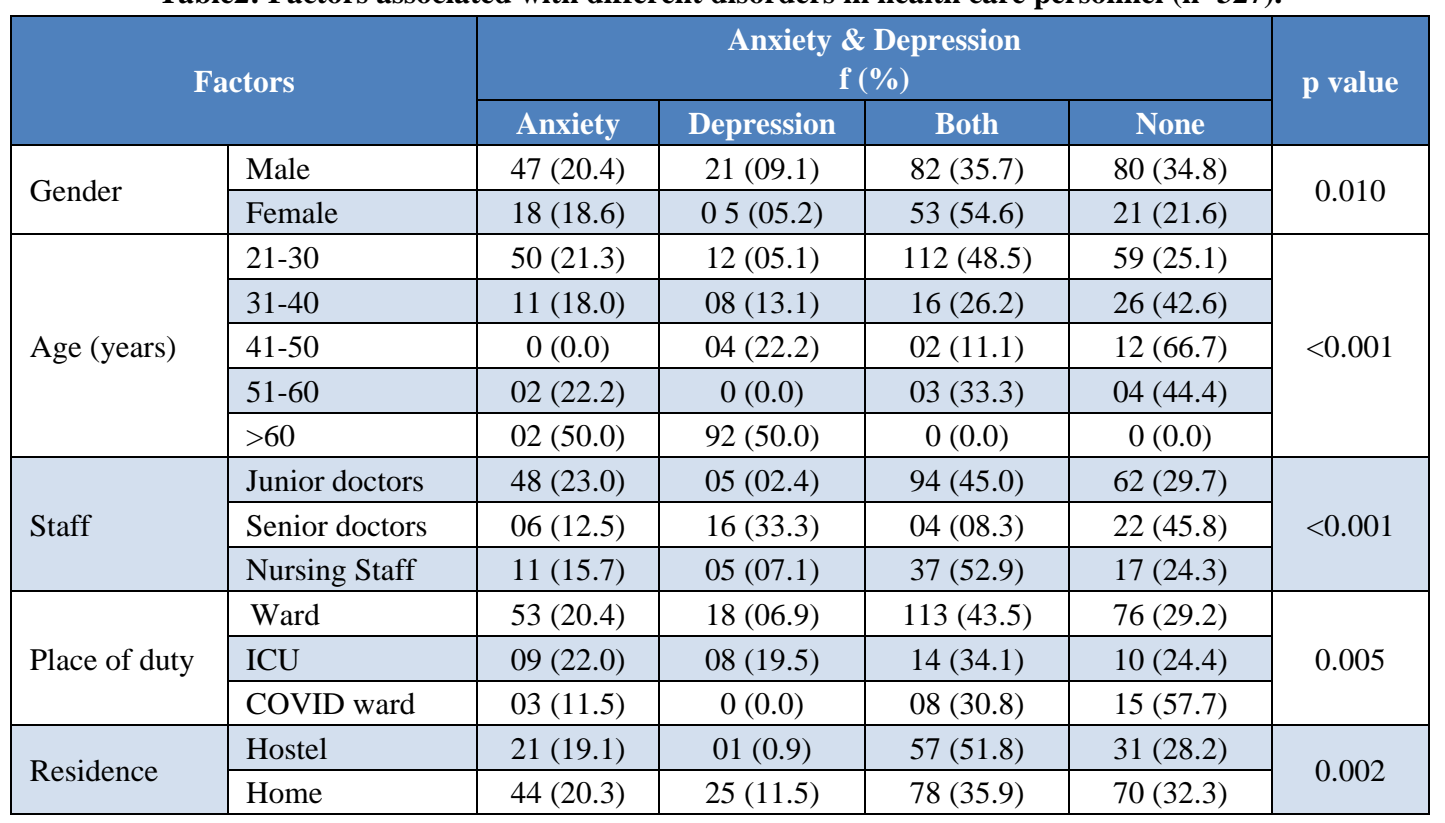

Disturbed mental status was significantly observed in female gender ( $p$ 0.01), 21-30 years age group and $>60 \mathrm{yrs}$ were affected more $(\mathrm{p}<0.001)$. Nursing staff had the highest number of affected ( $\mathrm{p}<0.001)$. Health care workers in ICU showed significantly higher number of mental disturbance than other places ( $p$ 0.005). The health care workers residing in Hostel reported more disorders than Day Scholars (p 0.02).

\section{DISCUSSION}

Among the 226(69.1\%) health care workers suffering from disturbed mental status, anxiety was more common than depression. This finding is consistent with other studies. The frequency of anxiety disorder was found to be more than the depressive illness in multiple Chinese studies, being $35.6 \%$ vs $19.8 \%,{ }^{14} 24.1 \%$ vs $13.5 \%,{ }^{13}$ and $25.5 \%$ vs $12.1 \%$. Tan et a ${ }^{15}$ from Singapore also described anxiety in $14.5 \% \mathrm{HCW}$ and depression in $8.9 \%$.

Female gender was more prone to get stress related mental disturbance. Our study also showed higher percentage of females suffering from disturbed mental status. In a study from China, $85 \%$ of mental distress was seen in females. ${ }^{13}$ Females had significantly higher anxiety ( $\mathrm{p} 0.003)$ and depression ( $\mathrm{p}$ 0.001) scores than males as described in a study by Salman et al. ${ }^{16}$ Females exhibited higher rates of affective symptoms compared to males in a systematic review by Pap $\mathrm{S}$ et al. ${ }^{9}$

Majority of disorders was seen in younger age group (21-30yrs) in our study. Liang et al. (2020) described that medical staff at younger age $(<30$ years) had higher self-rated depression scores than those with older age (30 years). ${ }^{17}$ The stress related disorder was more common in young aged HCWs. ${ }^{2}$ Likewise, $64.7 \%$ HCWs who suffered stress disorders were aged less than 40 years, as reported from China. ${ }^{18}$
In the previous SARS epidemic, higher percentage of paramedical staff presented with stress related mental disturbance than doctors. ${ }^{19,20}$ Likewise in our study, nurses were affected more than doctors, similar to other studies where nurses exhibited higher rate of stress related mental disturbance. ${ }^{9,13}$ In a large study in China, $60.8 \%$ of HCWs suffering from mental disturbance were nurses. ${ }^{18}$ The reason for this can be their close contact with patients and procedures.

Literature states that the health care workers in departments of Chest Medicine, Emergency Medicine, and Intensive Care Units were twice more likely to suffer from anxiety and depressive disorders due to high risk of getting infection. The exposure with aerosol generating procedures and respiratory secretion make them vulnerable to infection. ${ }^{4} \mathrm{~A}$ study from Pakistan has observed that medical staff of COVID-19 ICUs had statistically significant higher rate of anxiety disorder than HCWs working in other wards. ${ }^{16} \mathrm{We}$ also found that HCWs working in ICU were suffering from anxiety / depression symptoms.

HCWs availing hostel facility showed high magnitude of mental health disturbance. This aspect has not been described before in the literature to the best of our knowledge. The reason for this can be the lack of family support and fear of contracting disease in between roommates.

HCWs managing COVID-19 patients are at high risk of acquiring infection themselves but also carry this risk to their families. More so, the fact that the outcome of disease is poor in Black and Asian race, it may also add up to increased stress related psychological issues. Other than the fear of disease, the change in routine practice, workload, extra timings, shortage of Personal Protective Equipment (PPE) and sometimes affected quality of care also impose considerable challenges during pandemic. This issue remained neglected throughout the time of crisis, but still 
now when the pandemic in our area is going to fade, considerable number of HCWs must have been stressed. It is imperative to take measures to identify those at risk and to ensure timely support, care and intervention wherever needed.

\section{CONCLUSION}

In keeping with expectations, COVID-19 related psychological disturbances significantly affected healthcare staff of a tertiary care hospital of Peshawar, being more evident in females, younger age groups, nurses, and ICU staff on duty.

\section{LIMITATIONS}

Being a single center study, the findings may not be generalizable, though it is expected that other tertiary care hospitals, nationally and internationally, would be facing similar scenarios in the face of the COVID-19 pandemic. Time constraints and altered duties did not allow random sampling that would have been more representative.

\section{REFERENCES}

1. United Nations Sustainable Development Group. Policy Brief: COVID-19 and the need for action on mental health. 2020 May 13. [cited 2020 August 5]. Available from: https://unsdg.un.org/resources/policybrief-covid-19-and-need-action-mentalhealth.

2. Jansson M, Rello J. Mental health in healthcare workers and the Covid-19 pandemic era: Novel challenge for critical care. J Intensive \& Crit Care. 2020;6(2):6.

3. Lee SM, Kang WS, Cho AR, Kim T, Park JK. Psychological impact of the 2015 MERS outbreak on hospital workers and quarantined hemodialysis patients. Compr Psychiatry. 2018;87:123-7.

4. Lua W, Wang H, Linc Y, Li L. Psychological status of medical workforce during the COVID-19 pandemic: A crosssectional study. Psychiatry Research. 2020:288;112936.

5. Novel coronavirus (2019-nCoV) situation reports. [cited 2020 August 7]. Available from: https://www.who.int/emergencies/ diseases/novel-coronavirus-2019/situationreports

6. Ali S, Noreen S, Farooq I, Bugshan A, Vohra F. Risk Assessment of healthcare workers at the frontline against COVID-19. Pak J Med Sci. 2020:36(COVID19S4):COVID-19 Supplement 2020.

7. Amnesty International. Global: Amnesty analysis reveals over 7,000 health workers have died from COVID-19. [Internet]. Posted September 03, 2020. [cited 2020 September 05] Available from: https://www.amnesty.org/en/latest/news/2 020/09/amnesty-analysis-7000-healthworkers-have-died-from-covid19/.

8. Jamal S. COVID-19: 58 medical workers die fighting coronavirus in Pakistan. July
02, 2020. World Asia. Available from: https://gulfnews.com/world/asia/pakistan/c ovid-19-58-medical-workers-die-fightingcoronavirus-in-pakistan-1.72373593.

9. Pappaa S, Ntellac V, Giannakasc T, Giannakoulisc VG, Papoutsic E, Katsaounou P. Prevalence of depression, anxiety, and insomnia among healthcare workers during the COVID-19 pandemic: A systematic review and meta-analysis. Brain Behav Immun. 2020 Aug;88:901-7.

10. Kang L, Li Y, Hu S, Chen M, Yang C, Yang BX, et al. The mental health of medical workers in Wuhan, China dealing with the 2019 novel coronavirus. [online]. The Lancet Psychiatry. 2020 Mar 01;7(3):E14. [cited 2020 September 05]. Available from: https://www.thelancet.com/action/showPd f?pii=S2215-0366\%2820\%2930047-X.

11. Dewa CS, Loong D, Bonato $\mathrm{S}$, Trojanowski L. The relationship between physician burnout and quality of healthcare in terms of safety and acceptability: a systematic review. BMJ Open 2017;7:e015141.

12. Grace MK, VanHeuvelen JS. Occupational variation in burnout among medical staff: Evidence for the stress of higher status. Soc Sci Med. 2019;232:199-208.

13. Zhu Z, Xu S, Wang H, Liu Z, Wu J, Li G, et al. COVID-19 in Wuhan: Sociodemographic characteristics and hospital support measures associated with the immediate psychological impact on healthcare workers. [online article]. EClinicalMedicine. 2020 Jul 01;24:100443. [cited 2020 September 10]. Available from: https://www.thelancet.com/action/showPd f?pii=S2589-5370\%2820\%2930187-5.
14. Huanga Y, Zhaoa N. Generalized anxiety disorder, depressive symptoms and sleep quality during COVID-19 outbreak in China: a web-based cross-sectional survey. Psychiatry Research. 2020;288:112954.

15. Tan BY, Chew NW, Lee GK, Jing M, Goh Y, Yeo LL, et al. Psychological impact of the COVID-19 pandemic on health care workers in Singapore. [Letter]. Ann Intern Med. 2020 Apr 6:M20-1083.

16. Salman M, Raza MH, Mustafa ZU, Khan TM, Asif N, Tahir $\mathrm{H}$, et al. The psychological effects of COVID-19 on frontline healthcare workers and how they are coping: a web-based, cross-sectional study from Pakistan. Medrxiv.2020;

doi: https://doi.org/10.1101/2020.06.03.20 119867.

17. Liang Y, Chen M, Zheng X, Liu J. Screening for Chinese medical staff mental health by SDS and SAS during the outbreak of COVID-19. J Psychosom Res. 2020;133:1101-2.

18. J Lai, S Ma, Y Wang, Cai Z, Hu J, Wei N, et al. Factors associated with mental health outcomes among health care workers exposed to coronavirus disease 2019. JAMA Network Open. 2020 Mar 23;3:Article e203976.

19. Goulia P, Mantas C, Dimitroula D, Mantis D, Hyphantis T. General hospital staff worries, perceived sufficiency of information and associated psychological distress during the $\mathrm{A} / \mathrm{H} 1 \mathrm{~N} 1$ influenza pandemic. BMC Infect Dis. 2010;10:322.

20. Tam CW, Pang EP, Lam LC, Chiu HF. Severe acute respiratory syndrome (SARS) in Hong Kong in 2003: stress and psychological impact among frontline healthcare workers. Psychol Med. 2004 Oct;34(7):1197-204 